In the workshops, 11 patients and 130 nurses participated. Eligible adult patients, diagnosed with a rheumatic disease, were recruited by newsletter from the Dutch Arthritis Foundation. Nurses were recruited by newsletter from the Dutch Nurses Organisation (V\&VN).

According to participants, the framework alone is only useable when additional explanation and illustration of concepts will be provided and following missing topics are added; communication between specialisms, knowledge of the healthcare system, responsibility allocation, faith, religion, culture, nutrition, lifestyle, prevention

The self-management web appeared to be helpful. Not all cards with needs could be placed in this web. It was suggested to add following topics to the web: Peer support or experience experts' contact, handling treatment recommendations, patient empowerment, defining limitations and supporting services like physiotherapy and municipality.

There is overlap between topics of the web: Lifestyle, leisure and self-care. It was suggested to place associated topics together or give the same colour.

Practical ideas for application of the web and about involving an experienced expert were discussed as well as the role of health professionals. Integration in e-health, linked to the medical file with visual support is preferred. Patients have to prepare themselves for consulting the nurse or doctor.

Communication plays a very important role for all elements. The tool should be usable for people with limited health literacy skills and nurses need skills like motivational interviewing for using the tool.

Conclusion: Existing frameworks seem useful as a scientific basis for the development of a communication tool for self-management support. Usability of a draft tool will be explored in a pilot study.

References:

[1] Ammerlaan J. Preferences and needs of patients with a rheumatic disease regarding the structure and content of online self-management support. Pat Educ Counsel. 2017;100(3):501-8.

[2]Been-Dahmen, J.M.J. (2018). Self-Management Support: A broader perspective on what patients need and nurses could provide.

Disclosure of Interests: A.J.L. Meier: None declared, Yvonne van Eijk-Hustings Grant/research support from: grand from sanofi and UCB, Consultant of: fee from amgen, Bertha Maat: None declared

DOI: 10.1136/annrheumdis-2020-eular.4437

\section{FRI0627-HPR COGNITIVE DISORDERS IN SYSTEMIC LUPUS ERYTHEMATOSUS WITH JUVENILE-ONSET: ONE SINGLE CENTER EXPERIENCE}

M. Kaleda ${ }^{1}$, N. Stepanenko ${ }^{1}$, I. Nikishina ${ }^{1} .{ }^{1}$ V.A. Nasonova Research Institute of Rheumatology, Pediatric Department, Moscow, Russian Federation

Background: Juvenile-onset SLE (jSLE) is a more aggressive disease than in adults due to several reasons including cognitive dysfunction, significantly affected the compliance and social rehabilitation of patients (pts).

Objectives: To analyze the cognitive, emotional and communicative status of pts with jSLE.

Methods: The study included 31 pts (90.3\% girls) with jSLE, verified in accordance to SLICC criteria 2012. All pts underwent standard examination in accordance with the diagnosis; in case of suspected neuropsychiatric disorders pts were examined by a neurologist, psychiatrist. Classifying of neuropsychiatric disorders was performed in accordance with the recommendations of the ACR,1999. SLEDAI $2 \mathrm{~K}$ was used for disease activity assessment. All pts were examined by a clinical psychologist using the standard pathodiagnostic testing.

Results: The median age at the onset was 12.0 y.o. [10.6;14.5]. The median disease duration at the time of diagnosis -0.75 ys $[0.5 ; 2.1]$. $35.5 \%$ pts had neuropsychic disorders at the onset: psychoses $-12.9 \%$, headaches $-12.9 \%$, cognitive disorders $-19.4 \%$, mood disorders $-16.1 \%$, distal polyneuropathy $-12.9 \%$. MRI of the brain was performed in 15 pts: CNS vasculitis was diagnosed in 3 pts (2 with psychosis, 1 - with cognitive impairment). Median disease activity by SLEDAI at the time of diagnosis was 15 scores [10;23].

At the time of examination by the clinical psychologist, the median age of pts was 15.2 ys [12.9;16.5]. The median disease duration was 1.1 ys [0.6;3.8]. Cognitive disorders were detected in $96.8 \%$ of the pts. The auditory-speech short-term memory was distributed between the medium and high levels $(54.8 \%$ and $45.2 \%$, respectively), and the high level of memorization prevailed in the long-term memory $(67.7 \%$ high, $32.3 \%$ medium). A high level of indirect memory was revealed in $67.8 \%$ of pts, medium - in $25.8 \%$, and low - in $6.4 \%$. Distribution of the difficulties of attention were identified $(64.5 \%$ - uneven distribution, $35.5 \%$ - sufficient distribution), as well as increased attrition of attention $(74.2 \%$ - attrition is detected, $25.8 \%$ - no attrition). $58.1 \%$ of pts demonstrated a high level of inclusion into work, $41.9 \%$ - a low one. Concentration of attention was recognized as sufficient in $87.1 \%$ of pts, insufficient in $12.9 \%$. The effectiveness of attention was rated as good in $87.1 \%$, decreased - in $12.9 \%$; stability is sufficient - in $64.5 \%$, low - in $35.5 \%$. In the operational side of thinking, a decrease in the level of generalization was revealed (48.4\%); there were no disturbances in the motivational component, lability of thinking in the dynamic (12.9\%). Various neurotic fears are characteristic for $54.8 \%$ of pts; the level of personal anxiety was increased in $41.9 \%$, moderate - in $48.4 \%$, low - in $9.7 \%$. Signs of aggression were revealed in $19.4 \%$ of pts, a decrease in the level of social adaptation - in $51.6 \%$. Communication difficulties experienced $83.9 \%$ of pts. According to the results of the clinical conversation, attention was focused on availability of conflict situations with peers in the disease onset in $38.7 \%$ of pts.

Conclusion: Cognitive disorders were detected in the majority of pts with jSLE, regardless of the presence of neuropsychiatric disorders at the onset. The revealed features of the clinical and psychological status of pts with jSLE must be considered when working out an individual rehabilitation model and develop psycho-correctional programs.

Disclosure of Interests: None declared

DOI: 10.1136/annrheumdis-2020-eular.4237

\section{FRI0628-HPR EVALUATING A COMPLEX PACKAGE OF CARE IN THE EAST-MIDLANDS KNEE PAIN FEASIBILITY COHORT RANDOMISED CONTROLLED TRIAL}

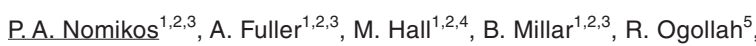
M. Doherty ${ }^{1,2,3}$, R. Nair ${ }^{2,6,7}$, D. Walsh ${ }^{1,2,3}$, A. Valdes ${ }^{1,2,3}$, A. Abhishek ${ }^{1,2,3}$. ${ }^{1}$ NIHR Nottingham Biomedical Research Centre, Nottingham, United Kingdom; ${ }^{2}$ Pain Centre Versus Arthritis, University of Nottingham, Nottingham, United Kingdom; ${ }^{3}$ Academic Rheumatology, University of Nottingham, Nottingham, United Kingdom; ${ }^{4}$ School of Health Sciences, University of Nottingham, Nottingham, United Kingdom; ${ }^{5}$ Nottingham CTU, University of Nottingham, Nottingham, United Kingdom; ${ }^{6}$ Institute of Mental Health, University of Nottingham, Nottingham, United Kingdom; ${ }^{7}$ Division of Psychiatry \& Applied Psychology, University of Nottingham, Nottingham, United Kingdom

Background: The role of nurses in managing painful knee OA has been advocated but whether nurses can deliver such interventions as a package of care is unknown. The overall aim of this research is to develop and test a nurse-led complex intervention for knee pain comprising non-pharmacological and pharmacological components. In the first study phase, we report on fidelity and acceptability of a non-pharmacological intervention, to resolve possible challenges to delivery.

Objectives: To evaluate fidelity of delivery and acceptability of non-pharmacological components of a complex intervention.

Methods: This was a mixed-methods study. Participants with chronic knee pain were recruited from the community to receive the intervention, delivered in 4-sessions over a 5-week period by a trained research nurse. The intervention consisted of holistic assessment, patient education and advice, aerobic and strengthening exercise and weight-loss advice if required. All sessions were video-recorded. Fidelity checklists were completed by the nurse (nurse-rated) and two researchers from the video-recordings (video-rated). Median fidelity scores (\%) and interquartile ranges (IQR) were calculated for each component and each session. Semi-structured interviews were conducted with participants. These were audio recorded, transcribed and analysed following the framework approach.

Results: 18 participants (34\% women), with a mean (SD) age and BMI of 68.7 (9.0) years and $31.2(8.4) \mathrm{kg} / \mathrm{m}^{2}$, took part in the study. Of these, 14 completed all visits. In total, 62 intervention sessions were assessed for fidelity. Overall fidelity was rated high by both nurse-rated scores $(97.7 \%)$ and video-rated scores $(84.2 \%)$. The level of agreement between nurse-rated and video-recorded methods was $73.3 \%(\mathrm{Cl} 71.3,75.3)$ and the inter-rater agreement was $65.5 \%(\mathrm{Cl} 60.3$, 70.5). Fidelity of delivery was lower for advice on footwear modification and walking aids in all sessions and moderate for education in session 1 and for exercise in session 4 (Table 1).

Table 1. Fidelity scores of the components of the intervention for each session,

\begin{tabular}{lcccc}
\hline Intervention components & Session $1^{*}$ & Session $2^{*}$ & Session $3^{*}$ & Session $4^{*}$ \\
\hline Education & $78.1(74.1,93.7)$ & $87.5(50,100)$ & $87.5(50,100)$ & $100(93.7,100)$ \\
Exercise & $94.4(88.9,100)$ & $88.9(75,94)$ & $86.1(72,100)$ & $75(67.6,82.8)$ \\
Adjunctive treatments & $50(45.83,100)$ & $0(0,50)$ & $50(0,100)$ & - \\
\hline
\end{tabular}

*median (IQR)

17 participants were interviewed. Most found advice supplied straightforward. They were satisfied with the package, which changed their perception of 